Trauma Berufskrankh 2014 • 16[Suppl 2]:202-205 DOI 10.1007/s10039-013-2011-0

Online publiziert: 16. Oktober 2013

(c) Springer-Verlag Berlin Heidelberg 2013

W. Barthlen · A. Kühn · C. Müller · D. Großmann

Kinderchirurgie, Universitätsmedizin Greifswald

\title{
Verletzte Kinder nach frischen Frakturen
}

\section{Erfordernis einer speziellen Rehabilitation?}

Heute bezieht also eine signifikante Anzahl erwachsener Personen eine Rente aufgrund einer im Kindesalter erlittenen Extremitätenfraktur. Gewiss sind begleitende Gefäß- oder Nervenverletzungen, eine Gelenkbeteiligung oder eine knöcherne Konsolidierung in Fehlstellung in erster Linie für das Ausbleiben einer vollständigen Restitutio ad integrum verantwortlich zu machen. Doch es ist auch nicht sichergestellt, dass im Heilungsverlauf alles Erdenkliche getan wurde, um eine Defektheilung zu vermeiden. In diesem Kontext stellt sich die Frage nach der Notwendigkeit einer spezifischen posttraumatischen Nachbehandlung im Kindesalter und ganz speziell nach dem Wert einer kindgerechten Physiotherapie.

\section{Spezifische posttraumatische Nachbehandlung im Kindesalter}

\section{Gegenargumente}

Ab dem 01.04.1971 wurde der Versicherungsschutz im Rahmen der DGUV auch auf Kindergarten- und Schulunfälle ausgedehnt. 40 Jahre später, im Jahr 2011, wiesen 10.633 Erwachsene eine MdE (Minderung der Erwerbsfähigkeit) in rentenberechtigendem Grad > 20\% aufgrund eines im Kindesalter erlittenen Unfalls in Schule oder Kindergarten auf. Bei 1650 Personen lag eine MdE von 50-100\% vor. In etwa 3200 Fällen waren Extremitätenverletzungen die Ursache, davon waren etwa 1500 Frakturen [pers. Mitteilung, Referat Statistik, DGUV (Deutsche Gesetzliche Unfallversicherung), 2013].
In kindertraumatologischen Lehrbüchern ist zur posttraumatischen Nachbehandlung im Kindesalter apodiktisch zu lesen:

„Grundsätzlich ist im Wachstumsalter keine medikamentöse, keine physikalische und keine physiotherapeutische Nachbehandlung frischer Frakturen und Luxationen notwendig" [7].

Begründende Literaturstellen stammen aus der Zeit zwischen 1957 und 1984. In anderen Werken ist dies vorsichtiger formuliert:
“... bedarf es beim Kind in der Regel keiner spezifischen Nachbehandlung ..." [10].

„Die regelmäßige Verordnung einer physiotherapeutischen Nachbehandlung ist im Kindesalter nur selten erforderlich und sinnvoll“ [10].

Zusätzlich wurde die Befürchtung geäuBert, dass eine Physiotherapie durch in der Behandlung von Kindern unerfahrene Therapeuten zu einer Übertherapie führen könnte [10]. Anhaltspunkte jedoch,

- wann von der Regel abgewichen werden sollte,

- bei welcher Indikation eine physiotherapeutische Nachbehandlung im Kindesalter sinnvoll sein könnte und

- welche Erkenntnisse die Furcht vor einer möglichen Übertherapie begründen,

wurden nicht gegeben. Diese Angst ist übrigens weltweit verbreitet: In einer Befragungsstudie aus Südkorea äußerten über 1/3 der Kindertraumatologen, dass eine posttraumatische Physiotherapie nach suprakondylärer Humerusfraktur im Kindesalter schädlich sein könnte [9].

\section{Mögliche Vorteile}

Eine fachgerechte physiotherapeutische Nachbehandlung könnte sich auf die Heilung verletzter Knochen, Bänder und Gelenkkapseln positiv auswirken. So könnte eine frühe aktive Bewegung die Gewebevitalität und Durchblutung fördern. 
Eine geringe Dehnung im Frakturbereich könnte die Zelldifferenzierung zu Kallusgewebe fördern. In der zunächst ungerichteten Proliferationsphase nach Verletzung ligamentärer Strukturen könnte durch eine vorsichtige Bewegung Adhäsionen vorgebeugt werden. Die Elastin- und Kollagenbildung könnten gefördert und durch die Ausrichtung der regenerierten Fasern gemäß der Belastung eine höhere Zugspannung und Gleitfähigkeit sowie einen größeren Bewegungsradius verletzter Sehnen- und Kapsel-BandStrukturen erreicht werden. Einer sekundären Einsteifung auch primär nicht betroffener benachbarter Gelenke sowie einer Muskelatrophie könnte entgegengewirkt werden [12].

Ein wichtiger zusätzlicher Aspekt könnte auch die Überwindung von kindlichen Ängsten sein. Kinder sind zwar keine kleinen Erwachsenen, sind aber in ihrem Charakter genauso vielfältig und verschieden wie alle Menschen. So gibt es Kinder, die sofort wieder aktiv sind, wenn der Gips abgenommen wurde. Es gibt aber auch das ängstliche Kind, das die bei der Fraktur erlittenen Schmerzen einfach nicht vergessen kann, über längere Zeit eine Schonhaltung einnimmt und Bewegungen meidet, obwohl die körperliche Heilung längst abgeschlossen ist.

\section{Erfahrungen der letzten 5 Jahre - Elternbefragung}

Um den Stellenwert einer posttraumatischen Physiotherapie im Kindsalter weiter zu untersuchen, versuchten wir, alle Familien von Kindern mit frischer Femurschaftfraktur, Unterschenkel- bzw. Tibiaschaftfraktur sowie suprakondylärer Humerusfraktur, die in den letzten 5 Jahren in der Klinik für Kinderchirurgie in Greifswald behandelt worden waren, telefonisch über ihre Erfahrungen zu befragen. Viele der bei uns behandelten Kinder befinden sich zurzeit des Unfalls im Urlaub oder auf Klassenfahrt und stammen aus der ganzen Republik. Die Nachsorge erfolgte daher in den meisten Fällen nicht bei uns, sondern heimatnah.

Wir empfahlen keine Physiotherapie für die weitere Behandlung und Nachsorge. Ausnahme waren suprakondyläre $\mathrm{Hu}$ merusfrakturen mit neurologischen Aus- fallerscheinungen. Wenn eine Physiotherapie durchgeführt wurde, war sie in der Regel durch den Hausarzt bzw. den weiterbehandelnden Arzt der heimatnahen Klinik verordnet worden, oft auch auf Drängen der Eltern.

Die Ergebnisse werden im Folgenden unabhängig von der Art - konservativ oder operativ - der Therapie für die Altersgruppe der 1 bis 5-jährigen Kindergartenkinder, der 6- bis 12-jährigen Schulkinder und der 13- bis 18-jährigen Adoleszenten angegeben.

\section{Femurfraktur}

In den Leitlinien der DGKiC (Deutsche Gesellschaft für Kinderchirurgie) zu Femurschaftfrakturen [2] von 2010 sind Physiotherapie oder Rehabilitation nicht erwähnt. Desgleichen sucht man auch in der Literatur, z. B. in einer großen Metaanalyse mit 2422 Fällen kindlicher Femurfrakturen [11], vergebens nach einem Hinweis. Demgegenüber mussten wir bei unserer Telefonrecherche feststellen, dass bei den Kindergartenkindern $(n=24)$ von 17 erreichten Kindern 4 eine Physiotherapie vom Hausarzt verschrieben bekommen hatten, 13 Kinder nicht. Bei den Schulkindern $(n=8)$ hatten von 6 erreichten Kindern 4 eine Physiotherapie erhalten und 2 nicht. In der Altersgruppe der Adoleszenten $(n=8)$ hatten von 6 erreichten Kindern 5 eine physiotherapeutische Nachbehandlung erhalten und nur 1 Kind nicht.

Alle Kinder zeigten nach telefonischer Auskunft ihrer Eltern einen vollen Bewegungsumfang der angrenzenden Hüftund Kniegelenke. Jeweils 1 Kind aus jeder Altersgruppe klagte über Wetterfühligkeit im Frakturbereich.

\section{Unterschenkel- bzw. Tibiafraktur}

Auch bei dieser Frakturlokalisation findet eine Physiotherapie im Rahmen der Nachbehandlung in den Leitlinien der DGKiC [1] von 2002 keine Erwähnung. Ebenso wenig fand das Thema Eingang in die Literatur. Eine Ausnahme stellt eine Studie aus Frankreich an 42 Kindern nach intramedullärer elastisch-stabiler Marknagelung (ESIN) dar, in welcher explizit ausgeführt wurde, dass eine Phy- siotherapie in der Nachbehandlung nicht verschrieben wurde [5]. Wie oft sie aber vielleicht dann doch durchgeführt wurde, bleibt offen. Zu unserer Überraschung fanden wir in Greifswald eine hohe Anzahl von nachträglich vom Hausarzt verschriebener Physiotherapie nach Unterschenkel- bzw. Tibiaschaftfraktur: 2 Kinder mit und 14 Kinder ohne Physiotherapie in der Kindergartenaltersklasse (insgesamt $n=17$ ), aber 10 mit und 17 ohne im Schulkindalter (insgesamt $n=33$ ). In der Adoleszentengruppe $(n=38)$ wurde 9-mal eine Physiotherapie durchgeführt, 23-mal nicht.

Auch hier wurden keine Einschränkungen im Bewegungsumfang benachbarter Gelenke angegeben. Wetterfühligkeit und Schmerzen im ehemaligen Frakturbereich nach starker Belastung wurden in Einzelfällen in beiden Nachbehandlungsgruppen erwähnt.

\section{Suprakondyläre Humerusfraktur}

In den Leitlinien der DGKiC [3] von 2010 heißt es bei den Empfehlungen zur Nachbehandlung dieses Frakturtyps bei konservativer Therapie:

\section{„Physiotherapie selten notwendig“ [3]}

und bei operativer Therapie

„Physiotherapie nur bei älteren Kindern mit anhaltender eingeschränkter Funktion". [3]

Eine kontrollierte randomisierte Studie jedoch, die suprakondyläre Humersufrakturen nach operativer Therapie in allen Schwere- und Dislokationsgraden, jedoch ohne neurovaskuläre Beteiligung, einschloss, kam zu dem Ergebnis, dass eine postoperative Physiotherapie bei diesen Frakturen im Kindesalter unnötig ist [6].

Es stellt sich also die Frage bezüglich der Evidenz. In einer bereits 2002 publizierten großen retrospektiven multizentrischen Studie, in deren Rahmen 540 Kinder (Erfassungsquote 61\%) nach suprakondylären Humerusfrakturen nachuntersucht wurden, ist zu lesen:

„Physiotherapie erwies sich grundsätzlich als unnötig und wurde dann verordnet, 
wenn die Ruhigstellungsdauer prolongiert war" [8].

In der gleichen Untersuchung jedoch wurden verbliebene Bewegungseinschränkungen (Flexionsdefizit $>10^{\circ}$, echtes Extensionsdefizit) für dritt- und viertgradige Frakturen in der Einteilung nach von Laer et al. [8] von 27\% angegeben. Zwar ist es fraglich, ob eine Physiotherapie in der Nachbehandlungszeit zu einer Verbesserung dieser unbefriedigenden Ergebnisse geführt hätte. Ob sie allerdings bei diesem hohen Anteil an verbliebenen Bewegungseinschränkungen so apodiktisch abgelehnt werden kann, ist ebenfalls fraglich.

Auch in Greifswald wurde von uns eine Physiotherapie nach suprakondylärer Humerusfraktur nur in Ausnahmefällen bei verbliebenen neurologischen Ausfällen verordnet. In unserem Telefoninterview ergab sich, dass in der Kindergartengruppe ( $n=25)$ von 23 auswertbaren Kindern 8 ohne Physiotherapie blieben, bei 5 Kindern wurde diese von den nachbehandelnden Kollegen verschrieben. Hier heilten alle Frakturen folgenlos aus. In der Schulkindgruppe jedoch $(\mathrm{n}=64)$ blieben 26 von 54 erreichbaren Kindern ohne Physiotherapie. Bei diesen gaben in 7 Fällen die Eltern eine verbliebene Funktionseinschränkung an („kann mit dem Arm nicht die gegenüberliegende Schulter erreichen"). Bei 28 Kindern jedoch wurde vom weiterbehandelnden Arzt eine Physiotherapie verschrieben, und bei 9 Kindern verblieb eine Einschränkung des Bewegungsumfangs.

Kinder im Adoleszentenalter mit suprakondylären Humerusfrakturen sahen wir erwartungsgemäß keine.

\section{Diskussion}

Natürlich lässt eine bloße Telefonbefragung von Eltern ohne eigene Untersuchung des Kindes nur sehr oberflächliche Schlussfolgerungen zu. Sicher sind unsere Zahlen zudem zu klein, um definitive Aussagen treffen zu können. Jedoch zeigte unsere kleine Studie eindeutig, dass bei Kindern nach Extremitätenfrakturen in beträchtlichem Umfang Physiotherapie entgegen der Empfehlung der erstbehandelnden Ärzte verschrieben wurde. Zeit-

Trauma Berufskrankh 2014 - 16[Suppl 2]:202-205 DOI 10.1007/s10039-013-2011-0

c) Springer-Verlag Berlin Heidelberg 2013

\section{W. Barthlen · A. Kühn · C. Müller · D. Großmann Verletzte Kinder nach frischen Frakturen. Erfordernis einer speziellen Rehabilitation?}

\section{Zusammenfassung}

Hintergrund. Bei einem Kind hat eine nach einem Unfall verbleibende Behinderung besonders gravierende Auswirkungen - nicht nur bezüglich der Kosten, sondern auch aufgrund der sich über die gesamte Lebenszeit erstreckenden Einschränkung der Lebensqualität.

Für und Wider der posttraumatischen Nachbehandlung im Kindesalter. Die noch in Lehrbüchern zu findende Aussage, dass im Wachstumsalter grundsätzlich keine medikamentöse, physikalische oder physiotherapeutische Nachbehandlung frischer Frakturen und Luxationen notwendig sei, basiert auf Ergebnissen aus der Zeit zwischen 1957 und 1984. Andererseits könnte sich eine fachgerechte physiotherapeutische Nachbehandlung positiv auf die Heilung verletzter Knochen, Bänder und Gelenkkapseln auswirken. Deshalb wurden die Eltern der in den letz- ten 5 Jahren in unserer Klinik wegen frischer Frakturen behandelten Kinder zur Nachbehandlung und zum Heilverlauf befragt. Dabei stellte sich heraus, dass vielen Kindern, obwohl vom erstbehandelnden Arzt nicht vorgesehen, vom nachbehandelnden Arzt eine Physiotherapie verschrieben wurde, von der sie nach Meinung der Eltern in der überwiegenden Zahl der Fälle auch profitierten. Resümee. Physiotherapie im Kindesalter sollte differenzierter betrachtet und klare Richtlinien und Indikationen nach den $5 \mathrm{~W}$ des Journalismus - wer, was, wo, wann, wie - erarbeitet werden. Sie sollte durch qualifizierte Kinderphysiotherapeuten durchgeführt werden.

\section{Schlüsselwörter}

Kinder - Knochenfraktur - Wachstumsalter . Nachbehandlung $\cdot$ Physiotherapie

\section{Injured children after fresh fractures. Is special rehabilitation necessary?}

\section{Abstract}

Background. In children who have had an accident, residual handicaps have a particularly serious effect, not only with respect to costs but also to the limitations which affect the quality of life over the whole life span. Pro and cons of posttraumatic follow-up treatment in childhood. The statement still to be found in textbooks that fundamentally no medicinal, physical or physiotherapeutic follow-up treatment of fresh fractures and dislocations is necessary in childhood is based on results from the time period from 1957 to 1984. On the other hand professionally performed physiotherapeutic follow-up treatment can have a positive effect on the healing of bones, tendons and joint capsules. For this reason, the parents of children who were treated for fresh fractures over the last 5 years in this hospital were surveyed on the follow-up treatment and healing process. The results showed that many children were prescribed physiotherapy by a second physician although this was not planned by the primary physician and according to the opinion of the parents in the majority of cases they profited from this.

Conclusions. Physiotherapy in childhood should be viewed in a differentiated manner and clear guidelines and indications should be worked out according to the five cardinal rules of who, what, where, when and how. The physiotherapy should be carried out by qualified pediatric physiotherapists.

\section{Keywords}

Children · Bone fractures - Growing age .

Rehabilitation · Physiotherapy punkt, Länge, Häufigkeit, beauftragter Therapeut und dessen Qualifikation jedoch differierten bei diesen Verordnungen. In den meisten Fällen waren es die Eltern selbst oder Familienangehörige, die den Anstoß zur Physiotherapie gegeben haben. Fast alle Eltern gaben im Telefoninterview an, dass die professionelle
Nachbehandlung ihrem Kind gut getan und geholfen hätte, die körperlichen und seelischen Traumafolgen zu überwinden. 


\section{Fazit für die Praxis}

- Es scheint klug, Physiotherapie im Kindesalter differenzierter zu betrachten und klare Richtlinien und Indikationen nach den $5 \mathrm{~W}$ des Journalismus zu erarbeiten: wer, was, wo, wann, wie?

- Physiotherapie im Kindesalter sollte von qualifizierten Kinderphysiotherapeuten durchgeführt werden.

- Die weit verbreitete Angst vor einer Übertherapie scheint schwer begründbar: Es ist kaum möglich, Kinder zu zwingen, schmerzhafte und für sie schädliche Bewegungen aktiv oder passiv durchzuführen. Wenn es dem Physiotherapeuten nicht gelingt, die Neugier des Kindes zu wecken und die gewünschten Bewegungsabläufe in spielerische Aktivitäten zu integrieren, wird er nichts ausrichten, aber auch kaum schaden können.

- Die Ergebnisse sollten in wissenschaftlichen Studien evaluiert werden, die in internationale Datenbanken wie PEDro („physiotherapy evidence database") Eingang finden können [4]. Auf diese Weise würde eine evidenzbasierte Beurteilung ermöglicht werden.

\section{Korrespondenzadresse}

\section{Prof. Dr. W. Barthlen}

Kinderchirurgie, Universitätsmedizin Greifswald, Sauerbruchstraße 1, 17475 Greifswald winfried.barthlen@uni-greifswald.de

\section{Einhaltung ethischer Richtlinien}

Interessenkonflikt. W. Barthlen, A. Kühn, C. Müller und D. Großmann geben an, dass kein Interessenskonflikt besteht.

Dieser Beitrag beinhaltet keine Studien an Menschen oder Tieren.

The supplement containing this article is not sponsored by industry.

\section{Literatur}

1. Deutsche Gesellschaft für Kinderchirurgie (2002) Unterschenkelschaftfraktur Tibiafraktur. AWMFLeitlinienregisternummer 006/060. AWMF, Düsseldorf. http://www.awmf.org/uploads/tx_szleitlinien/006-060.pdf. Zugegriffen: 07.07.2013
2. Deutsche Gesellschaft für Kinderchirurgie (2010) Femurschaftfraktur, AWMF-Leitlinienregisternummer 006/016. AWMF, Düsseldorf. http://www. awmf.org/uploads/tx_szleitlinien/006-016I-S1_Femurschaftfraktur.pdf. Zugegriffen: 07.07.2013

3. Deutsche Gesellschaft für Kinderchirurgie (2010) Suprakondyläre Humerusfraktur beim Kind. AWMF-Leitlinienregisternummer 012/014. AWMF Düsseldorf

4. Elkins MR, Moseley AM, Sherrington C et al (2013) Growth in the physiotherapy evidence database (PEDro) and use of the PEDro scale. Br J Sports Med 47:188-189

5. Griffet J, Leroux J, Boudjouraf N et al (2011) Elastic stable intramedullary nailing of tibial shaft fractures in children. J Child Orthop 5:297-304

6. Keppler P, Salem K, Schwarting B et al (2005) The effectiveness of physiotherapy after operative treatment of supracondylar humeral fractures in children. J Pediatr Orthop 25:314-316

7. Laer L von (2007) Frakturen und Luxationen im Wachstumsalter, 7. Aufl. Thieme, Stuttgart New York

8. Laer L von, Günter SM, Knopf S et al (2002) Die suprakondyläre Oberarmfraktur im Kindesalter eine Effizienzstudie Ergebnisse der multizentrischen Studie der Sektion Kindertraumatologie der Deutschen Gesellschaft für Unfallchirurgie - Teil II: Aufwand und Nutzen der Behandlung. Unfallchirurg 105:217-223

9. Lee S, Park MS, Chung CY et al (2012) Consensus and different perspectives on treatment of supracondylar fractures of the humerus in children. Clin Orthop Surg 4:91-97

10. Marzi I (2009) Kindertraumatologie, 2. Aufl. Springer, Berlin Heidelberg New York

11. Poolman Rw, Kocher Ms, Bhandari M (2006) Pediatric femoral fractures: a systematic review of 2422 cases. J Orthop Trauma 20:648-654

12. Senn E (1999) Physikalische Therapie - Krankengymnastik und passiv-physikalische Maßnahmen. In: Mutschler W, Haas N (Hrsg) Praxis der Unfallchirurgie. Thieme, Stuttgart New York, S 977-997 